\title{
Bevacizumab and risk of intracranial hemorrhage in patients with brain metastases: a meta-analysis
}

\author{
Lin Yang ${ }^{1} \cdot$ Chuan-Jie Chen ${ }^{2} \cdot$ Xiao-Ling Guo ${ }^{3}$ Xiao-Cui Wu ${ }^{1} \cdot$ Bo-Jie Lv ${ }^{4} \cdot$ Hong-Li Wang ${ }^{1} \cdot$ Zhi Guo $^{5}$. \\ Xiang-Yang Zhao ${ }^{6}$ (i)
}

Received: 18 August 2017 / Accepted: 18 November 2017 / Published online: 23 November 2017

(c) The Author(s) 2017. This article is an open access publication

\begin{abstract}
Administration of bevacizumab to patients with brain metastases (BM) is controversial due to concerns about the increased risk of intracranial hemorrhage (ICH). This meta-analysis assessed whether the risk of ICH increases in BM patients receiving treatments that contain bevacizumab versus without. PubMed, Embase, Cochrane Library and annual meeting abstracts of the American Society of Clinical Oncology up to 13 November 2016 were searched for studies that referred to ICH complications due to bevacizumab in patients with BM. Eight studies involving 8713 patients were included in this analysis. Compared with the control arm without bevacizumab, the bevacizumab treatment arm did not exhibit a significant increase in ICH [odds ratio (OR) 1.20; 95\% confidence intervals (CI) 0.69-2.09; $\mathrm{P}=0.53$ ]. Subgroup analyses with retrospective studies showed a similar result, although subgroup analyses with prospective studies failed. This meta-analysis revealed that bevacizumab does not significantly increase the risk of ICH in solid tumor patients with BM.
\end{abstract}

Keywords Bevacizumab $\cdot$ Brain metastases $\cdot$ Intracranial hemorrhage $\cdot$ Meta-analysis

Lin Yang and Chuan-Jie Chen have contributed equally to this article.

Xiang-Yang Zhao

46309407@qq.com

1 Department of Oncology, 266 Hospital, PLA, Pu-Ning Road, Shuangqiao District, Chengde 067000, China

2 Department of Orthopedics, Chengde Central Hospital, Second Affiliated Hospital of Chengde Medical University, 22 Xi-Da Street, Shuangqiao District, Chengde 067000, China

3 Department of Neurology, 266 Hospital, PLA, Pu-Ning Road, Shuangqiao District, Chengde 067000, China

4 Department of Scientific Research, 266 Hospital, PLA, Pu-Ning Road, Shuangqiao District, Chengde 067000, China

5 Department of Oncology, Shanxi Provincial Hospital of Traditional Chinese Medicine, 16 Bing-Zhou-Xi Street, Yingze District, Taiyuan 030001, China

6 Department of Surgical Oncology, 266 Hospital, PLA, $\mathrm{Pu}$-Ning Road, Shuangqiao District, Chengde 067000, China

\section{Introduction}

Brain metastases (BM) are the most common intracranial tumors in adult patients [1] and occur in up to $40 \%$ of adult cancer patients [2]. The prognosis of patients with BM is poor, with median overall survival ranging from weeks to months in untreated patients [3]. In adults, BM generally originate from primary lung (40-50\%), breast (15-25\%), renal, gastrointestinal tract tumors (4-6\%) and melanoma (5-20\%) [4]. The incidence of BM depends on the tumor type and molecular subtype [5], with melanoma having the highest incidence (approximately 50\%) [6]. The incidence of $\mathrm{BM}$ is rising for many reason, e.g., increasing occurrence of tumors prone to metastasis to the brain such as lung cancer, wide utilization of powerful imaging technologies such as magnetic resonance imaging during upfront staging and follow-up, longer survival of cancer patients due to earlier detection and better treatment, and the advent of novel therapeutic compounds with good anti-neoplastic activity but inadequate penetration through the blood-brain barrier [5, 7].

Bevacizumab is a recombinant, humanized monoclonal antibody targeting vascular endothelial growth factor (VEGF), which is a key factor associated with tumor 
angiogenesis and growth $[8,9]$. Bevacizumab was proven effective for diverse solid tumors, including metastatic breast cancer [10], non-squamous non-small cell lung cancer (NSCLC) [11, 12], colorectal cancer [13], renal cell carcinoma [14], and recurrent glioblastoma [15]. Until recently, patients with central nervous system (CNS) metastases have been routinely excluded from bevacizumab-containing clinical trials, following a single case in which a severe intracranial hemorrhage (ICH) from a previously undiagnosed brain metastasis was observed in a patient with hepatocellular carcinoma (HCC) during a phase I study of bevacizumab in 1997 [16]. The potential risk of ICH precludes the use of bevacizumab in solid tumor patients with BM [17]. Although several reports have recently concluded that there is no increased risk of ICH in BM patients receiving bevacizumab treatment $[8,18-21]$, application of bevacizumab in such patients remains controversial. To date, no meta-analysis of association between bevacizumab and ICH risk in patients with $\mathrm{BM}$ has been reported. In this study, we performed a meta-analysis to assess whether BM patients receiving treatments containing bevacizumab have a higher risk of ICH than patients receiving treatments without bevacizumab.

\section{Methods}

\section{Eligibility criteria}

Studies that met the following criteria were included: (1) Subjects were solid tumor patients with BM. (2) Experimental arm patients received chemotherapy/targeted therapy with bevacizumab, and control arm patients received the same chemotherapy/targeted therapy with or without placebo. (3) Data were available for ICH.

\section{Literature search strategy}

PubMed, Embase and Cochrane Library were comprehensively searched for studies that referred to ICH complications of bevacizumab in solid tumor patients with BM (data cutoff date: 13 November 2016). The annual meeting abstracts of the American Society of Clinical Oncology were also searched from inception of the database to June 2016. The following search terms were used: 'adverse effect', 'AE', 'safety', 'toxic*', 'side effect', 'bleed*', 'hemorrhag*', 'haemorrhag*', 'toleren*', 'cerebrovascular event', 'complication', 'bevacizumab', 'avastin', 'cancer', 'tumor', 'carcinoma' and 'neoplasm'. The search strategy is shown in Table 1. The search was limited to English publications in human subjects.

\section{Study selection}

Two reviewers independently performed the initial search, deleted duplicate records, reviewed the titles and abstracts for relevance, and identified each as exclude or requiring further assessment. If deemed necessary, the full text of the article was retrieved and reviewed in detail to identify eligible studies according to the predefined inclusion criteria. Discrepancies were resolved by consensus.

\section{Data extraction}

Two reviewers independently abstracted data, including the name of the first author, publication year, study design, indication, sample size of each arm, number of patients experiencing ICH in each arm, treatment regimens, bevacizumab treatment time, bevacizumab dose, BM status when patients enrolled in the primary study, and evaluation criteria for ICH. Again, discrepancies were resolved by consensus.

\section{Quality assessment}

Two reviewers independently evaluated the methodological quality of included studies according to the Newcastle-Ottawa Scale (NOS) [22]. The reviewers resolved disagreement by discussion.

\section{Statistical analysis}

Data were analyzed using RevMan 5 (http://ims.cochrance. $\mathrm{org} / \mathrm{revman}$ /download). Differences were expressed as odds ratios (ORs) with 95\% confidence intervals (CIs) for dichotomous outcomes. Heterogeneity across the included studies was evaluated by the Cochrane's Q-test $\mathrm{I}^{2}$ statistic.

Table 1 Search strategy

\begin{tabular}{ll}
\hline Search & Terms \\
\hline$\# 1$ & Bevacizumab OR avastin \\
$\# 2$ & Adverse effect OR AE OR safety OR toxic* OR side effect OR bleed* \\
& OR hemorrhag* OR haemorrhag* OR toleren* OR cerebrovascular \\
& event OR complication \\
$\# 3$ & Cancer OR tumor OR carcinoma OR neoplasm \\
$\# 4$ & $\# 1$ AND \#2 AND \#3 \\
\hline
\end{tabular}


$\mathrm{P}>0.1$ and $\mathrm{I}^{2}<50 \%$ indicated a lack of inter-study heterogeneity; $\mathrm{P}<0.1$ and $\mathrm{I}^{2}>50 \%$ indicated that the studies were heterogeneous [23], and we explored the causes of heterogeneity by subgroup, sensitivity, and other analyses. A fixed-effects model (Peto method) was used regardless of heterogeneity because ICH was a rare event in BM patients receiving medical therapy with or without bevacizumab (events with incidence $<1 \%$ were defined as rare events) [24]. Publication and selection bias were investigated through funnel plots. A two-sided $\mathrm{P}$ value $<0.05$ was considered statistically significant.

\section{Results}

\section{Study identification and selection}

Using our search strategy, a total of 26,466 records were retrieved from the initial database search. After excluding duplicate articles, 24,388 records remained. After a simple reading of the titles and abstracts of the articles, 24,276 records were removed, including articles not potentially relevant to the analysis, articles of primary CNS malignancies, review articles, meta-analyses, case reports or case series, studies without control arms, studies with both arms containing bevacizumab, studies with different regimens in different arms except bevacizumab or placebo, and studies containing other anti-VEGF drugs except bevacizumab. The remaining 112 full-text articles were reviewed in detail, and 104 of them were also removed because the studies did not include patients with BM, articles were written in another language, or the data for ICH was not available. Finally, a total of eight studies were included in this meta-analysis $[18,21,25-30]$. The selection process is shown in Fig. 1.

\section{Study characteristics}

The key characteristics of the included studies are summarized in Table 2. Eight studies involving 8713 patients were included in this analysis. Six studies were retrospective, and two were prospective. 8053 of the 8713 patients were included in the experimental group receiving bevacizumabcontaining treatment, and 660 were included in the control group receiving treatment without bevacizumab. Five of the eight included studies reported the dose of bevacizumab in bevacizumab-treated patients. Four out of eight studies had control arms in which patients received placebo. The highand all-grade ICH events were not differentiated when the study data were abstracted because very few studies reported all-grade or low-grade (grade 1-2) ICH.

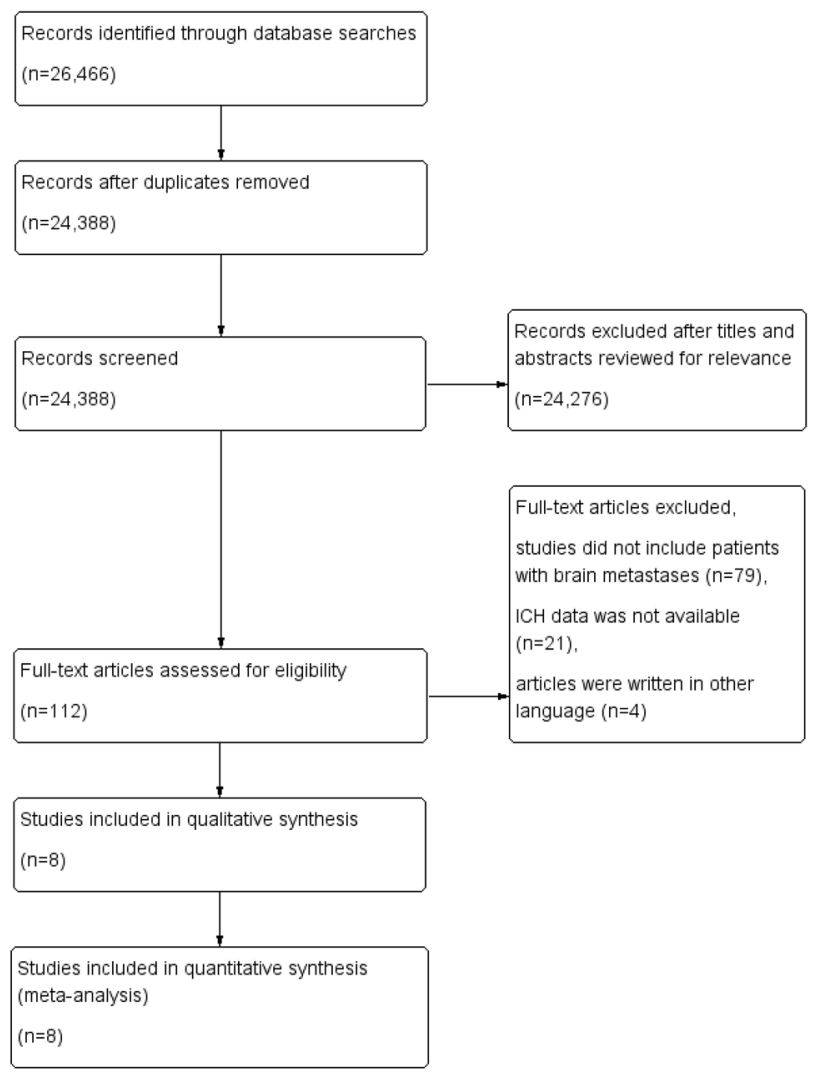

Fig. 1 Selection process for the studies included in the meta-analysis

\section{Quality assessment}

The NOS results are summarized in Table 3. Among the six retrospective studies, one received eight stars, three received seven stars, and two received six stars. One of two prospective studies received nine stars, and the other received eight stars.

\section{Risk of ICH}

Eight studies, totaling 8713 patients and 338 events, provided data on ICH. In four studies, neither the experimental arm nor control arm had patients who developed ICH [25-27, 30], ICH occurred in the experimental arm, but not the control arm in one study [18], and the remaining three studies had ICH patients in both arms [21, 28, 29]. There was no heterogeneity across the eight studies included in the analysis $\left(\mathrm{P}=0.60, \mathrm{I}^{2}=0 \%\right)$ despite definite differences, including primary tumor type, study design and bevacizumab dose. A fixed-effects model employing the Peto method was applied. Four studies in which no patients developed ICH in either the experimental arm or the control arm were excluded from meta-analysis by RevMan 5 during data processing, yielding results that were labeled "Not 


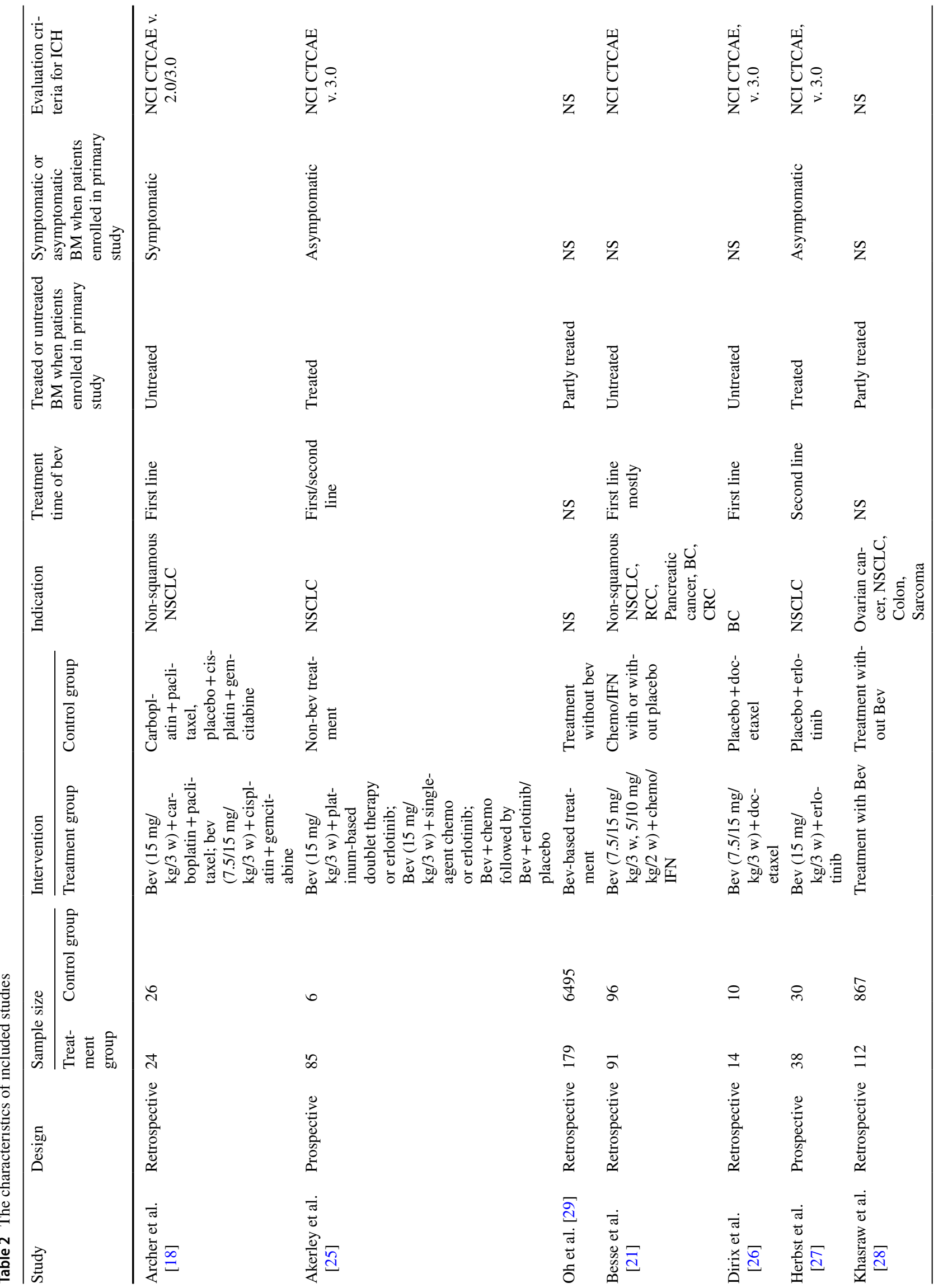


estimable" instead of producing an OR and CI. The pooled OR was 1.20 , with $95 \% \mathrm{CI}$ from 0.69 to $2.09(\mathrm{P}=0.53)$. This result indicates that no significant increase in $\mathrm{ICH}$ was found in the bevacizumab-containing treatment arm compared with that in the control arm (Fig. 2).

Subgroup analysis was performed to assess the influence of study design on the OR for ICH. The analysis of six retrospective studies, with a total of 8554 patients and 338 events, revealed that ICH was not significantly elevated in the bevacizumab arm compared with that in the control arm (OR 1.20; 95\% CI 0.69-2.09; $\mathrm{P}=0.53)$. However, the analysis of the prospective study group failed because neither the bevacizumab arm nor control arm had patients who developed ICH in the two prospective studies, and RevMan 5 produced results that were labeled "Not estimable" instead of producing an OR and CI (Fig. 2).

\section{Publication bias}

A funnel plot to evaluate publication bias requires at least ten studies to be included in the meta-analysis, otherwise, the test power will be too low to assess the symmetry of the funnel plot. However, the meta-analysis described here included only eight studies, and in four studies, neither the bevacizumab arm nor the control arm had patients who developed ICH. Therefore, we did not generate a funnel plot.

\section{Discussion}

This is the first meta-analysis to evaluate the risk of ICH in $\mathrm{BM}$ patients with versus without bevacizumab treatment. We found no significant difference in risk of ICH in solid tumor patients with BM based on bevacizumab treatment. Furthermore, the results were consistent in subgroup analysis of the retrospective studies, although subgroup analysis of the prospective studies failed because not enough prospective studies were identified, and no patients developed ICH in the two prospective studies that were included.

Notably, four studies in which neither the experimental arm nor the control arm contained patients who developed ICH were excluded from this meta-analysis by RevMan 5 during data processing, and the results of this meta-analysis were derived from the remaining four studies. However, no difference in the risk of ICH was detected in the patients receiving the bevacizumab-containing versus non-bevacizumab-containing treatment in the four studies excluded from analysis, although the sample sizes of the bevacizumab arm and control arm in each excluded study are quite different. Thus, the results of the four excluded studies are consistent with the meta-analysis results of the remaining four studies. In addition, the same situation was observed in subgroup analysis. 
Table 3 Results of quality assessment for studies using NOS

\begin{tabular}{llllll}
\hline Study & Study design & Selection & Comparability & Exposure & Outcome \\
\hline Oh et al. [29] & Case-control & $* * \mathrm{ab}$ & $* \mathrm{c}$ & $* * *$ & \\
Archer et al. [18] & Case-control & $* * * \mathrm{~b}$ & $* \mathrm{c}$ & $* * *$ & \\
Besse et al. [21] & Case-control & $* * \mathrm{ab}$ & $* *$ & $* * *$ \\
Dirix et al. [26] & Case-control & $* * * \mathrm{~b}$ & $* \mathrm{c}$ & $* * *$ & \\
Khasraw et al. [28] & Case-control & $* * \mathrm{ab}$ & $* \mathrm{c}$ & $* * *$ & \\
Tang et al. [30] & Case-control & $* * * \mathrm{~b}$ & $* *$ & & $* * *$ \\
Akerley et al. [25] & Cohort & $* * * *$ & $* *$ & & $* * *$ \\
Herbst et al. [27] & Cohort & $* * * *$ & $* \mathrm{c}$ & & \\
\hline
\end{tabular}

Reasons for lost stars: ${ }^{a}$ the case was defined by record linkage; ${ }^{b}$ hospital controls were selected; ${ }^{c}$ there was no description of whether the study controlled for any additional factor, such as gender or age

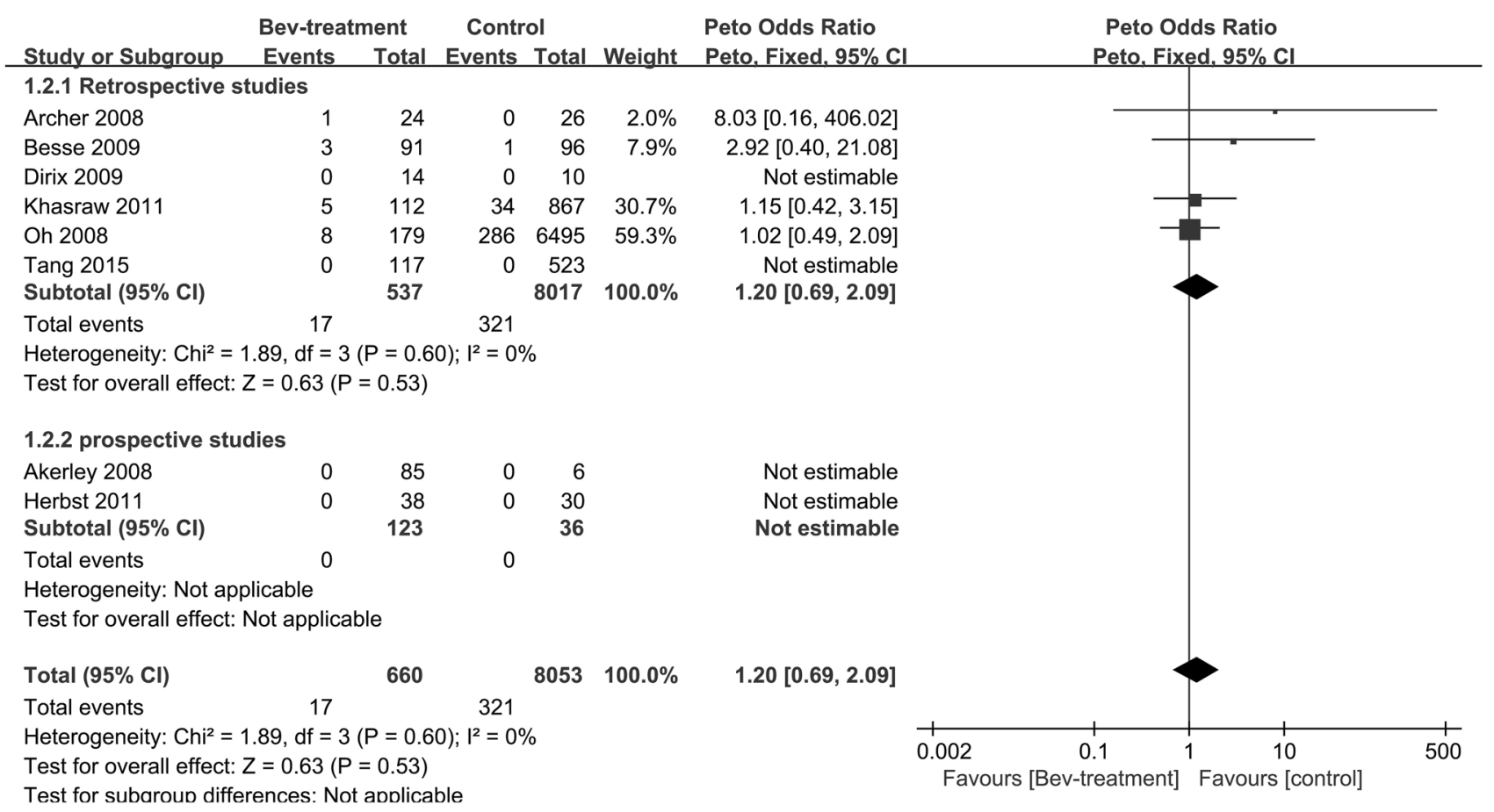

Fig. 2 Incidence of ICH in bevacizumab versus control group

In a phase II prospective noncomparative study (BRAIN, NCT00800202) investigating the efficacy and safety of bevacizumab in non-squamous NSCLC patients with asymptomatic untreated BM, only one of the 91 patients enrolled experienced an ICH event (grade 1) [31]. In another single-arm phase II trial (PASSPORT, AVF3752g) addressing bevacizumab safety in patients with non-squamous NSCLC and previously treated $\mathrm{BM}$, no grade $2 \mathrm{CNS}$ hemorrhages occurred [8]. The reported incidences of cerebral hemorrhage in patients with CNS metastases not exposed to bevacizumab range from 5 to $29 \%$ [21, 32-36]. Comparing these background ICH rates with those presented in the BRAIN and PASSPORT studies, there is no apparent increased risk of cerebral hemorrhage in bevacizumab-treated patients with
CNS metastasis, although direct cross-trial comparisons should be viewed with caution. These results are consistent with our findings presented in this meta-analysis.

Clinical trials containing bevacizumab still routinely exclude patients with CNS metastasis due to concerns about the increased risk of $\mathrm{ICH}$, arising partly from a single case in a phase I trial of bevacizumab, in which a 29 -year-old patient with HCC experienced a fatal cerebral hemorrhage from a previously undiagnosed brain metastasis in 1997 [16]. However, the risk for spontaneous bleeding of CNS metastases varies with the histology of the tumor, with a $<1$ or 5\% chance of occurrence in lung or breast BM, respectively, compared with significantly higher rates for metastases derived from thyroid cancer, melanoma (40-50\%), 
renal cell cancer (70\%), choriocarcinoma or HCC [37]. CNS metastases from $\mathrm{HCC}$ have an inherent propensity to hemorrhage because patients with $\mathrm{HCC}$ are likely to have coagulation disorders due to liver dysfunction, resulting in ICH incidences up to $87.5 \%$, independent of the type of therapy received [21, 38-41].

Our study has several limitations. First, patients with some factors, such as medications associated with bleeding (anticoagulants, etc.) [42], thrombocytopenia [42], tumor histology predisposing to bleeding [37], evidence of bleeding diathesis or coagulopathy [43], uncontrolled hypertension [44], or history of thrombotic or hemorrhagic disorders [44], are at risk of ICH intrinsically, and these factors are important stratified factors in evaluating whether bevacizumab increases the risk of ICH in patients with BM. However, limited stratified analysis of these factors was performed in this study because these factors were not reported in a portion of individual primary studies included in this meta-analysis. Second, the number of studies enrolled in this meta-analysis was small. Only eight studies met the eligibility criteria and were included in the analysis. Finally, not all the included studies were RCTs, which is the gold standard for clinical research and has less bias than other study designs.

In conclusion, this meta-analysis revealed that bevacizumab does not significantly increase the risk of ICH in solid tumor patients with $\mathrm{BM}$, and it has provided evidence indicating that $\mathrm{BM}$ patients with a low incidence of $\mathrm{ICH}$ [21], such as those with advanced/metastatic breast cancer, NSCLC, renal and colorectal cancer, may not be generally excluded from bevacizumab therapy or trials. It will be important to validate these findings in RCTs with larger cohorts.

Acknowledgements We thank Sandeep S. and Aimee Talarski for manuscript language editing.

\section{Compliance with ethical standards}

Conflict of interest The authors declare that they have no conflict of interest.

Research involving human participants This article does not contain any studies with human participants performed by any of the authors.

Open Access This article is distributed under the terms of the Creative Commons Attribution 4.0 International License (http://creativecommons.org/licenses/by/4.0/), which permits unrestricted use, distribution, and reproduction in any medium, provided you give appropriate credit to the original author(s) and the source, provide a link to the Creative Commons license, and indicate if changes were made.

\section{References}

1. Gavrilovic IT, Posner JB (2005) Brain metastases: epidemiology and pathophysiology. J Neurooncol 75(1):5-14

2. Nayak L, Lee EQ, Wen PY (2012) Epidemiology of brain metastases. Curr Oncol Rep 14(1):48-54

3. Preusser M, Capper D, Ilhan-Mutlu A, Berghoff AS, Birner P, Bartsch R et al (2012) Brain metastases: pathobiology and emerging targeted therapies. Acta Neuropathol 123(2):205-222

4. Fidler IJ (2011) The role of the organ microenvironment in brain metastasis. Semin Cancer Biol 21(2):107-112

5. Caffo M, Barresi V, Caruso G, Cutugno M, La Fata G, Venza M et al (2013) Innovative therapeutic strategies in the treatment of brain metastases. Int J Mol Sci 14(1):2135-2174

6. Amer MH, Al-Sarraf M, Baker LH, Vaitkevicius VK (1978) Malignant melanoma and central nervous system metastases: incidence, diagnosis, treatment and survival. Cancer 42(2):660-668

7. Owonikoko TK, Arbiser J, Zelnak A, Shu HK, Shim H, Robin AM et al (2014) Current approaches to the treatment of metastatic brain tumours. Nat Rev Clin Oncol 11(4):203-222

8. Socinski MA, Langer CJ, Huang JE, Kolb MM, Compton P, Wang L et al (2009) Safety of bevacizumab in patients with non-small-cell lung cancer and brain metastases. J Clin Oncol 27(31):5255-5261

9. Shih T, Lindley C (2006) Bevacizumab: an angiogenesis inhibitor for the treatment of solid malignancies. Clin Ther 28(11):1779-1802

10. Miller K, Wang M, Gralow J, Dickler M, Cobleigh M, Perez EA et al (2007) Paclitaxel plus bevacizumab versus paclitaxel alone for metastatic breast cancer. N Engl J Med 357(26):2666-2676

11. Johnson DH, Fehrenbacher L, Novotny WF, Herbst RS, Nemunaitis JJ, Jablons DM et al (2004) Randomized phase II trial comparing bevacizumab plus carboplatin and paclitaxel with carboplatin and paclitaxel alone in previously untreated locally advanced or metastatic non-small-cell lung cancer. J Clin Oncol 22(11):2184-2191

12. Sandler A, Gray R, Perry MC, Brahmer J, Schiller JH, Dowlati A et al (2006) Paclitaxel-carboplatin alone or with bevacizumab for non-small-cell lung cancer. N Engl J Med 355(24):2542-2550

13. Hurwitz H, Fehrenbacher L, Novotny W, Cartwright T, Hainsworth J, Heim W et al (2004) Bevacizumab plus irinotecan, fluorouracil, and leucovorin for metastatic colorectal cancer. N Engl J Med 350(23):2335-2342

14. Escudier B, Pluzanska A, Koralewski P, Ravaud A, Bracarda S, Szczylik C et al (2007) Bevacizumab plus interferon alfa-2a for treatment of metastatic renal cell carcinoma: a randomised, double-blind phase III trial. Lancet 370(9605):2103-2111

15. Friedman HS, Prados MD, Wen PY, Mikkelsen T, Schiff D, Abrey LE et al (2009) Bevacizumab alone and in combination with irinotecan in recurrent glioblastoma. J Clin Oncol 27(28):4733-4740

16. Gordon MS, Margolin K, Talpaz M, Sledge GW Jr, Holmgren E, Benjamin R et al (2001) Phase I safety and pharmacokinetic study of recombinant human anti-vascular endothelial growth factor in patients with advanced cancer. J Clin Oncol 19(3):843-850

17. Kuse N, Yoshimori K, Ueyama M, Hirukawa I, Nakagawa Y, Rina I et al (2012) Safety of bevacizumab-containing chemotherapy in non-small-cell lung cancer patients with brain metastases. Ann Cancer Res Ther 20(2):47-51

18. Archer V, Reck M, Sandler AB, Johnson DH, Kong G, Strickland DK et al (2008) Risk of symptomatic central nervous system (CNS) progression and secondary hemorrhage in patients with non-squamous non-small cell lung cancer (NSCLC) receiving bevacizumab (BV)-based first-line therapy. J Clin Oncol 26(15_Suppl):8114- 
19. Morgensztern D, Govindan R (2008) Treatment of patients excluded from Eastern Cooperative Oncology Group 4599 and AVAiL studies: focus on brain metastasis and squamous histology. Clin Lung Cancer 9(Suppl 2):S57-S61

20. Oh Y, Stewart DJ (2008) Systemic therapy for lung cancer brain metastases: a rationale for clinical trials. Oncology (Williston Park) 22(2) 168-178 (discussion 78, 83, 88 passim)

21. Besse B, Lasserre SF, Compton P, Huang J, Augustus S, Rohr UP (2010) Bevacizumab safety in patients with central nervous system metastases. Clin Cancer Res 16(1):269-278

22. Wells GA, Shea BJ, O'Connell D, Peterson J, Welch V, Losos M et al (2012) The Newcastle-Ottawa Scale (NOS) for assessing the quality of non-randomized studies in meta-analysis. Appl Eng Agric 18(6):727-734

23. Li Q, Yan H, Zhao P, Yang Y, Cao B (2015) Efficacy and safety of bevacizumab combined with chemotherapy for managing metastatic breast cancer: a meta-analysis of randomized controlled trials. Sci Rep 5:15746

24. Bradburn MJ, Deeks JJ, Berlin JA, Russell LA (2007) Much ado about nothing: a comparison of the performance of meta-analytical methods with rare events. Stat Med 26(1):53

25. Akerley WL, Langer CJ, Oh Y, Strickland DK, Royer SJ, Xia Q et al (2008) Acceptable safety of bevacizumab therapy in patients with brain metastases due to non-small cell lung cancer. J Clin Oncol 26(15_Suppl):8043-

26. Dirix LY, Romieu G, Provencher L, Grimes D, Viana LDS, Paterson A et al (2009) Safety of bevacizumab (BV) plus docetaxel (D) in patients (pts) with locally recurrent (LR) or metastatic breast cancer ( $\mathrm{mBC}$ ) who developed brain metastases during the AVADO phase III study. Can Res 69(2 Suppl):4116

27. Herbst RS, Ansari R, Bustin F, Flynn P, Hart L, Otterson GA et al (2011) Efficacy of bevacizumab plus erlotinib versus erlotinib alone in advanced non-small-cell lung cancer after failure of standard first-line chemotherapy (BeTa): a double-blind, placebocontrolled, phase 3 trial. Lancet 377(9780):1846-1854

28. Khasraw M, Holodny A, Goldlust SA, DeAngelis LM (2012) Intracranial hemorrhage in patients with cancer treated with bevacizumab: the Memorial Sloan-Kettering experience. Ann Oncol 23(2):458-463

29. Oh Y, Wallace S, Taylor S, Herbst RS, Lippman SM, Karp DD et al (2008) Minimally increased risk of cerebrovascular occlusive disease or intracerebral hemorrhage in patients on bevacizumab treatment and association with intracerebral malignancies. J Clin Oncol 26(15_Suppl):14507-

30. Tang N, Guo J, Zhang Q, Wang Y, Wang Z (2016) Greater efficacy of chemotherapy plus bevacizumab compared to chemo- and targeted therapy alone on non-small cell lung cancer patients with brain metastasis. Oncotarget 7(3):3635-3644
31. Besse B, Le Moulec S, Mazieres J, Senellart H, Barlesi F, Chouaid $\mathrm{C}$ et al (2015) Bevacizumab in patients with nonsquamous nonsmall cell lung cancer and asymptomatic, untreated brain metastases (brain): a nonrandomized, phase ii study. Clin Cancer Res 21(8):1896-1903

32. Byrne TN, Cascino TL, Posner JB (1983) Brain metastasis from melanoma. J Neurooncol 1(4):313-317

33. Lieu AS, Hwang SL, Howng SL, Chai CY (1999) Brain tumors with hemorrhage. J Formos Med Assoc 98(5):365-367

34. Maiuri F, D'Andrea F, Gallicchio B, Carandente M (1985) Intracranial hemorrhages in metastatic brain tumors. J Neurosurg Sci 29(1):37-41

35. Mandybur TI (1977) Intracranial hemorrhage caused by metastatic tumors. Neurology 27(7):650-655

36. Mathieu D, Kondziolka D, Cooper PB, Flickinger JC, Niranjan A, Agarwala $S$ et al (2007) Gamma knife radiosurgery in the management of malignant melanoma brain metastases. Neurosurgery 60(3):471-481 (discussion 481-482)

37. Carden CP, Larkin JM, Rosenthal MA (2008) What is the risk of intracranial bleeding during anti-VEGF therapy? Neuro Oncol 10(4):624-630

38. Choi HJ, Cho BC, Sohn JH, Shin SJ, Kim SH, Kim JH et al (2009) Brain metastases from hepatocellular carcinoma: prognostic factors and outcome: brain metastasis from HCC. J Neurooncol 91(3):307-313

39. Chang L, Chen YL, Kao MC (2004) Intracranial metastasis of hepatocellular carcinoma: review of 45 cases. Surg Neurol 62(2):172-177

40. Murakami K, Nawano S, Moriyama N, Sekiguchi R, Satake M, Fujimoto H et al (1996) Intracranial metastases of hepatocellular carcinoma: CT and MRI. Neuroradiology 38(Suppl 1):S31-S35

41. Seinfeld J, Wagner AS, Kleinschmidt-DeMasters BK (2006) Brain metastases from hepatocellular carcinoma in US patients. J Neurooncol 76(1):93-98

42. Letarte N, Bressler LR, Villano JL (2013) Bevacizumab and central nervous system (CNS) hemorrhage. Cancer Chemother Pharmacol 71(6):1561-1565

43. Robert NJ, Dieras V, Glaspy J, Brufsky AM, Bondarenko I, Lipatov ON et al (2011) RIBBON-1: randomized, double-blind, placebo-controlled, phase III trial of chemotherapy with or without bevacizumab for first-line treatment of human epidermal growth factor receptor 2-negative, locally recurrent or metastatic breast cancer. J Clin Oncol 29(10):1252-1260

44. Reck M, von Pawel J, Zatloukal P, Ramlau R, Gorbounova V, Hirsh V et al (2009) Phase III trial of cisplatin plus gemcitabine with either placebo or bevacizumab as first-line therapy for nonsquamous non-small-cell lung cancer: AVAil. J Clin Oncol 27(8):1227-1234 